\title{
Time-Frequency Analysis Based on PNN for Nonstationary Random Vibration of Spacecraft
}

\author{
Hai Yang ${ }^{1}$ Wei Cheng ${ }^{1}$ Hong Zhu ${ }^{2}$
}

${ }^{1}$ Institute of Solid Mechanics, Beijing University of Aeronautics and Astronautics, Beijing 100083, P. R. China

${ }^{2}$ Liao Ning Equippment Manufacture College of Vocational Technology, Shenyang 110034, P. R. China

\begin{abstract}
In view of the disadvantages of the traditional time-varying algorithm about nonstationary random vibration signal of a spacecraft with close spaced modal frequency. A process neural network (PNN) based on the empirical mode decomposition (EMD) method is put forward. First, the EMD method is utilized to decompose the signal into several intrinsic mode functions (IMFs). Then for each IMF, The PNN is established and time-varying auto-spectral density is obtained. Finally, the time-varying auto-spectral density of the signal can be reconstituted by linear superposing. The example results show the effectiveness of this new method in time-frequency analysis.
\end{abstract}

Keywords: Time-varying parameter, Power spectrum, Process neural network, EMD

\section{Introduce}

The traditional analytical method of power spectrum about random vibration signal is usually supposed that the signal is stable. However the vibration of spacecraft during the flight is nonstationary. To deal with this vibration, we usually use time-frequency analytical methods, Such as short-time Fourier transform, Wigner-Ville distribution, wavelet analysis etc[1]. These methods give a exact description of the time-varying character to nonstationary signals at some degree and make up the deficiency of traditional methods, but some limits are also exposed, such as fake signal and fake frequency. The method of Hilbert-Huang transform (HHT) brought forward by Norden E. Huang has made a breakthrough in dealing with nonstationary signals[2]. The core of the method is that it can decompose the complex datum into some intrinsic mode functions (IMFs) self-adaptively. And each IMF has definite physical content by empirical mode decomposition (EMD). Compared with small-wave method, this is a self-adaptive time-frequency analytical method which needs no knowledge stored .The basis function of the EMD only depend on the signal and the decomposition has definite physical content. Although HHT has been brought forward for a short time, the theory and the application have been caught much attention and have been researched widely[3].

The nonstationary signal sampled by spacecrafts during the flight has the character of close frequency, low-signal-to-noise and complex-waves. If the signal is decomposed directly by EMD, the standard IMF (at a time corresponding to only one frequency) can not be obtained, and a multi-component IMF signal should be obtained. If the Hilbert spectrum has been analyzed with the nonstandard IMF directly, the time-frequency distribution obtained is fault. The article[4] has made some analysis to the question mentioned above and has given some corresponding criterions, but those criterions have some limit during the application. To resolve the question mentioned above, PNN is used to make time-varying analysis to the nonstandard IMF of the real vibration signal during the spacecraft flight. So an exact time-varying auto-spectral density should be obtained by the method and the distinguish rate of frequency is very high.

\section{Empirical Mode Decomposition}

The EMD is a method of decomposing a signal in the time domain. The decomposition is based on the direct extraction of the signal energy associated with various intrinsic modes in different time scales. The EMD method is developed from the simple assumption that any signal consists of different simple intrinsic modes of oscillations. Each linear or nonlinear mode will have the same number of extrema and zero-crossings. There is only one extremum between successive zero-crossings. Each mode should be independent of the others. In this way, each signal could be decomposed into a number of IMFs, each must satisfy the following definition[5]: (1) the number of zero-crossings and the number of extrema in whole sampled datum must either be equal or differ at most by one;(2) at any point of the datum, the mean value of the envelope 
defined by the local maxima and the envelope defined by the local minima is zero. An IMF represents a simple oscillatory mode as a counter part of the simple harmonic function, but it allows the modulation of amplitude and frequency. Therefore it is much more generic than other signal processing methods.

With the definition of IMF, any signal can be simply decomposed by its local maxima and minima. Once the extrema of the signal are identified, all the local maxima can be connected by a cubic spline line as the upper envelope. For local minima, repeating the procedure, the lower envelope can be obtained. The mean of the upper and lower envelopes is designated as $m_{1}(t)$, and the difference between the original data $x(t)$ and $m_{1}(t)$ is the first component, $h_{1}(t)$, i.e.,

$$
x(t)-m_{1}(t)=h_{1}(t) .
$$

Ideally, $h_{1}(t)$ should be an IMF since the construction of $h_{1}(t)$ described above has satisfied all the requirements of IMF. If $h_{1}(t)$ does not meet the definition of a true IMF, the sifting process has to repeat many times until a true IMF is obtained. In the following sifting process, $h_{1}(t)$ is treated as the data to be processed. Then

$$
h_{1}(t)-m_{11}(t)=h_{11}(t),
$$

where $m_{11}(t)$ is the mean of upper and lower cubic spines and fits the maxima and minima of the signal $h_{1}(t), h_{11}(t)$ is the subsequent trial IMF. The sifting process can repeat $k(k=1,2,3 \cdots)$ times until $h_{1 k}(t)$ is a true IMF, that is

$$
h_{1(k-1)}(t)-m_{1 k}(t)=h_{1 k}(t) .
$$

Then the first IMF component of the signal can be designated as

$$
c_{1}(t)=h_{1 k}(t)
$$

$c_{1}(t)$ should contain the finest scale or the shortest period component of the signal. We can separate $c_{1}(t)$ from the rest of the data by

$$
x(t)-c_{1}(t)=r_{1}(t) .
$$

Since the residue still contains long period components, it should be treated as the new data and subject to the same sifting process as described above. This procedure can repeat for all the subsequent $r_{j}(t)(j=1,2, \cdots, n-1)$ and the result is

$$
\begin{gathered}
r_{1}(t)-c_{2}(t)=r_{2}(t) \\
r_{2}(t)-c_{3}(t)=r_{3}(t) \\
\vdots \\
r_{n-1}(t)-c_{n}(t)=r_{n}(t) .
\end{gathered}
$$

The sifting process can stop by any of the following predetermined criteria: either when the component $c_{n}$ or the residue $r_{n}$ becomes so small and less than the predetermined value of substantial consequence, or when the residue $r_{n}$ becomes a monotonic function and no another IMF can be extracted from it. By summing up equation (5) and (6) , we finally represent the original signal in the following equation

$$
x(t)=\sum_{i=1}^{n} c_{i}+r_{n}
$$

which is decomposed into $n$-empirical modes, and a residue $r_{n}$, which can be either a monotonic function or single cycle. To apply the EMD, a mean or zero reference is not required; EMD only needs the locations of the local extrema.The sifting process generates the zero reference for each component, except for the residue. The components of the EMD are usually physically meaningful, because the characteristic scales are defined by the physical data themselves.

Unlike the frequency filtering methods that cause deformation of the signal filtered if it is either nonlinear or nonstationary, a time-space filter can be devised for signal reconstruction using the IMFs. The EMD filter can preserve the full nonlinearity and nonstationary in the physical space since a nonlinear and nonstationary signal generates harmonics of all ranges. For example, the output of a low-pass filter used for a signal having $n$ IMF components can expressed simply as

$$
x_{l k}(t)=\sum_{j=k}^{n} c_{j}(t)+r_{n}(t)
$$

The output of a high-pass filter can be expressed as

$$
x_{h k}(t)=\sum_{j=1}^{k} c_{j}(t)
$$

and the output of a band-pass filter can be expressed as

$$
x_{b k}=\sum_{j=b}^{k} c_{j}(k) .
$$

Where $b$ and $k$ are the number of the selected intermediate modes. A band-cut filter may be designed simply by omitting the selected components in the reconstruction progress. Hence the EMD method is a self-adaptive method of dealing with signals. It is fit to nonstationary process. However the decomposition has applied cubic spline interpolation, some serious distortion appears at the end of signal. If the distortion is too serious, the latter decomposition will lose research value. That is named end effect, which has been discussed in some articles[6]. It is impossible to decompose according 
to the IMF definition strictly during the actual process, thus the IMFs obtained are not ideal intrinsic mode functions. To solve the question, a criterion is needed to judge the rationality. Now the applied widely criterion is the standard difference of the former and latter $h(t)$, i.e.

$$
s d=\sum_{t=0}^{T}\left(\frac{h_{i(k-1)}(t)-h_{i(k)}(t)}{h_{i(k-1)}(t)}\right)^{2} .
$$

$s d$ usually take the value from 0.2 to 0.3 . In equation (11), the subscript $i$ denotes the $i_{\text {th }}$ order IMF component, $k$ denote times of repetition, $T$ denote total time of dispersed signal sequence.

Having obtained the IMF components, we have no difficulties in applying the Hilbert transform to calculate a meaningful instantaneous frequency for each component. Hence, the original data can be expressed as

$$
X(\omega, t)=\operatorname{Re} \sum_{i=1}^{n} a_{i}(t) e^{i \int \omega_{i}(t) d t},
$$

where $a_{i}(t)$ is a time-dependent expansion coefficient and the argument of the exponential $\omega_{i}(t)$ is the instantaneous frequency of each component as a function of time respectively, and the residue $r_{n}$ is omitted. Comparing the equation with Fourier transform, the IMF represents a more general expansion with variable amplitudes and instantaneous frequencies. The EMD improves greatly not only the efficiency, but also makes it able to accommodate nonlinear and nonstationary signals.

\section{Process Neural Network}

\subsection{Network structure}

To some complex signals with closely spaced frequencies, there are some errors between IMF components obtained by decomposition and true components at a certain, because the value of $s d$ depend on experience greatly. Thus It is difficult to decompose it as independent IMF component by EMD. Usually The first order IMF is a signal of wide band frequency and mono-component. The obtained time-frequency distribution is different from the fact if the IMF is analyzed by Hilbert spectrum. The method of Short Time Fourier Transform is incapable to the matter if it considers time precision. Though wavelet transform can make decomposition by selection of appropriate scale, much trashy harmonic produced synchronously. In order to debase the difference above, PNN is used to make time-varying parameter analysis to IMF in this paper. Aimed at the problems of the time-varying information processing and the dynamic system modeling, a kind of model with time-varying inputs and outputs function was built. In the PNN with time-varying inputs and outputs function, the time accumulation operator of process neuron is adopted as the integral to time or other algebra operations, its space-time aggregation mechanism and incitation can synchronously reflect the space aggregation and stage time accumulation effect of exterior time-varying input signals to the output results, so as to complete the complex mapping relationship between the inputs and outputs of non-linear system[7].

It is supposed that the structure of PNN is arranged in layers with $p-M_{1}-M_{2}-1$. Namely, There are $p$ neurons in input-layer, which are used to input $p$ time-varying signals; The first hidden layer is used to input projection of discrete time series under the primary function $b_{L}(t)$; The first hidden layer is made of $M_{1}$ time-varying process neurons, which are adopted as the integral to time or other algebra operations; The second hidden layer is made of $M_{2}$ non- time-varying common neurons, which are used to improve the mapping ability of network to complex relation between inputs and outputs. The fourth layer is output-layer and is used to complete output of the system. The structure of PNN is shown as Fig.1.

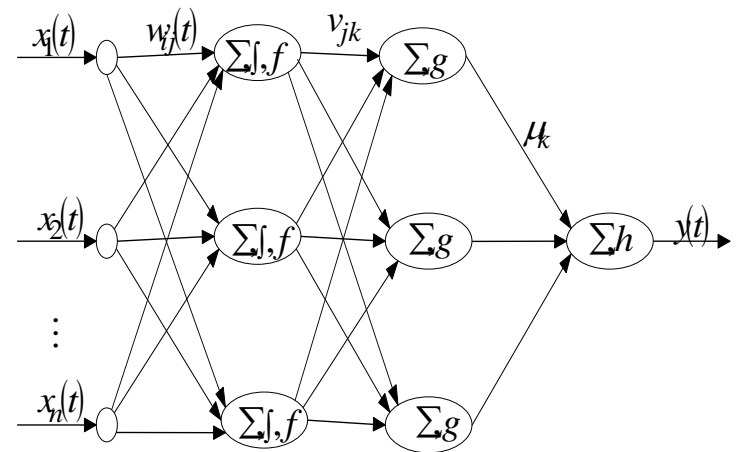

Fig.1:Structure of four layer PPN

In Fig.1, if the input space of PNN is [0 T]; the discrete time series $\{x(n-1), \cdots x(n-p)\}$ denote the input function. $\left\{w_{i j}(t)\right\}$ is the connection weighting value from input-layer node $i$ to hidden layer node $j ; \theta_{j}^{1}$ is the energizing threshold of the first hidden layer node $j ; f$ is energizing function. So the output of the first hidden layer node $j$ can be obtained commonly as follows:

$$
y_{j}^{1}=f\left(\sum_{i=1}^{n} \int_{0}^{t} w_{i j}(\tau) x_{i}(t) d \tau-\theta_{j}^{1}\right) .
$$


Depend on the output of the first hidden layer, the output of the second hidden layer can be stated as follows:

$$
y_{k}^{2}=g\left(\sum_{j=1}^{m} v_{j k} y_{j}^{1}-\theta_{k}^{2}\right) .
$$

$\left\{v_{j k}\right\}$ is the connection weighting value from the first hidden layer node $j$ to the second hidden layer node $k ; \theta_{k}^{2}$ is the energizing threshold of the second hidden layer node $k ; g$ is energizing function.

So depend on the output of the second hidden layer, the output of the network can be stated as follows:

$$
\begin{aligned}
y(t)= & h\left(\sum _ { k = 1 } ^ { L } \mu _ { k } g \left(\sum _ { j = 1 } ^ { m } v _ { j k } f \left(\sum_{i=1}^{n} \int_{0}^{t} w_{i j}(\tau) x_{i}(t) d \tau\right.\right.\right. \\
& \left.\left.\left.-\theta_{j}^{1}\right)-\theta_{k}^{2}\right)-\theta^{3}\right)
\end{aligned}
$$

$\left\{u_{k}\right\}$ is the connection weighting value from the second hidden layer node $k$ to output-layer node; $\theta^{3}$ is the energizing threshold of the out-layer node; $h$ is energizing function.

If all the threshold value is zero and energizing functions are linear, Equation (15) can be simplified as follows:

$$
y(t)=\sum_{k=1}^{L} \mu_{k} \sum_{j=1}^{m} v_{j k} \sum_{i=1}^{n} \int_{0}^{t} w_{i j}(\tau) x_{i}(t) d \tau .
$$

If the input space of PNN is [0 $\left[\begin{array}{l}0\end{array}\right]$ and $b_{1}(t), b_{2}(t), \cdots, b_{R}(t)$ are a group standard orthogonal basis functions; weighting value $w_{i j}(\tau)$ is functions about time, for the convenience of calculation; Weighting functions are expanded in the form of basis functions in the same group:

$$
w_{i j}(\tau)=\sum_{r=1}^{R} w_{i j}^{r} b_{r}(\tau) .
$$

$w_{i j}^{r}$ is the connection weighting from input node $i$ to the first hidden layer node $j$ relative to $b_{r}(\tau)$. Replacing the $w_{i j}(\tau)$ in equation(16) using the relationship from equation(17) yields

$$
y(t)=\sum_{i=1}^{n} \sum_{k=1}^{L} \sum_{j=1}^{m} \mu_{k} v_{j k} \int_{0}^{t} \sum_{r=1}^{R} w_{i j}^{r} b_{r}(\tau) x_{i}(t) d \tau .
$$

Then, $a_{i}(t)$ is designated as

$$
a_{i}(t)=-\sum_{k=1}^{L} \sum_{j=1}^{m} \mu_{k} v_{j k} \int_{0}^{t} \sum_{r=1}^{R} w_{i j}^{r} b_{r}(\tau) d \tau,
$$

and $y(t)$ can be written in the useful form

$$
y(t)=-\sum_{i=1}^{n} a_{i}(t) x_{i}(t) .
$$

If the expected output of network is $d(t)$ and the error is $\varepsilon(t)$, then

$$
d(t)=y(t)+\varepsilon(t)=-\sum_{i=1}^{n} a_{i}(t) x_{i}(t)+\varepsilon(t) .
$$

Equation (21) can be stated as $n$ order time-varying $A R$ model.

Mapping the input signal to the time-varying basis functions and trained by the PNN, the time-varying parameter of $A R$ model is feasible to be calculated by the connection weighting value obtained after network reaching stabilization. A time-varying power spectrum of the signal can be obtained according to the transfer function of $A R$ model and the inputted white noise[8], which can be stated as follows:

$$
s(f, t)=\frac{\Delta t \sigma_{p}^{2}(t)}{\left|1+\sum_{k=1}^{P} a_{k}(t) \exp (-j 2 \pi k f \Delta t)\right|^{2}}
$$

In the equation, $\sigma_{p}^{2}(t)$ is the average power of the energizing white noise $(W(t))$ of the time-varying $A R$ model. In addition, $W(t)$ is the error-output $\varepsilon(t)$ of the PNN. It is no difficult to estimate the $\sigma_{p}^{2}(t)$ by PNN.

\subsection{Order identification}

When $A R$ model is used to approach a random signal, the order of the model should be selected appropriately. Generally, the order of the $A R$ model is not known at the beginning. If the order is selected too low, the power spectral will be too smooth, which will lower the distinguish rate of frequency; If the order is selected too high, there will produce fake frequency. The AIC criterion, showed by Akaike, is now generally used in stationary random signal, which can be stated as follows:

$$
A I C(k)=N \log \sigma_{k}^{2}+2 k \text {. }
$$

Where $N$ is the number of the data sample, $\sigma_{k}$ is the predicted error of the model. For models assumed under different order, the order corresponded to the minimum of the AIC is the appropriate order. For the nostationary random signal, KonzinF[9] analyzed the sufficient condition and researched the application of the AIC criterion in identifying the order of the nostationary random signal model. He found that these problems and parameter estimations were uniform in progressive normal state. So in this paper, the AIC criterion was chosen to identify the order of the time-varying model.

\subsection{Training algorithm}


The input of PNN and the connection weighting can both be the functions of time. A converge operator of time is added to process neuron, so the training of PNN is very different from the other common neural networks. The calculation of PNN is more complicated. Meanwhile, because the form of network connection weighting and the parameter contained are random, the connection weighting function is very hard to be confirmed by the training, during which the learning sampling basis used has no common form of functions.

A group of proper basis functions is brought in input space. Input functions are transformed into limited series according to the basis function by given precision. Meanwhile, network weighting functions are expressed as the expanded forms in the same group of basis functions, which can simplify the complexity of process neuron about time-aggregation operation. According to this algorithm, there are the same complexity between learning PNN and training traditional feed forward neural network. The application shows that this algorithm has not only simplified the operation of PNN but also increased the stability and convergence in network learning. There are many other methods to choose orthogonal basis function. Basis function can be chosen as Circular function, Multinomial function, Fourier basis function, Wavelet basis function, Walsh function etc.

Based on the predigesting algorithm mechanism, the algorithm of group training is employed to complete the calculation of errors. If there are $P$ groups of training samplings, according to the gradient descent algorithm, the error in Fig. 1 is obtained as:

$$
E(k)=\frac{1}{P} \sum_{j=1}^{P}\left(y_{j}^{2}-d_{j}^{2}(k)\right)^{2} .
$$

In (24), $y_{j}$ is the output of the $j_{t h}$ group training sample; $d_{j}(k)$ is the expected output of $k_{\text {th }}$ training.

Traditional gradient decent algorithm has some flaws in lower convergence speed and smallest in part etc, in practical applications. By simulation, "false saturation" and heavy oscillation are proved to happen often on networks, so it is very hard to obtain satisfying training results.

Adding momentum into the regulation of weighting can not only rise the convergence speed of network, but also prevent the oscillation when error curved surface is regulated. The regulation equation is

$$
\Delta w(k+1)=\eta \Delta w(k)+\alpha(1-\eta) h(k),
$$

$$
\begin{aligned}
w(k+1) & =w(k)+\Delta w(k+1), \\
h(k) & =\frac{\partial E(k)}{\partial w(k)},
\end{aligned}
$$

where $\alpha(0<\alpha<1)$ is learning speed, and $\eta(0<\eta<1)$ is regulation coefficient of momentum, $w(k)$ is network weighting value of every layer.

If error $E<\xi$ or training times $K<N_{\max }$ the expectation of network has been achieved. Then output the learning results and end training, save network weighting value and error regulated every time. Otherwise, the value of circulation time added 1 , and then the regulation is continued.

\section{Case study}

Thirteen orders IMF components and a residue $r$ were obtained by the EMD decomposition to the nonstationary random vibration signals of a spacecraft sampled during the flight. The first four orders IMF components and the residue were shown in fig2.

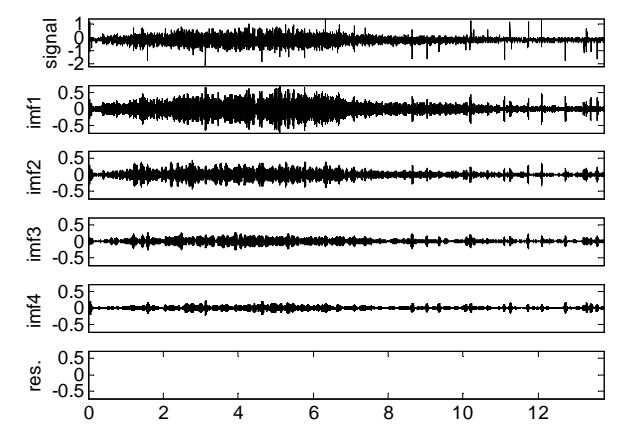

Fig.2 :origin signal, the first four orders IMF components and the surplus item.

It was shown that each order IMF component contained different time rule which made the character of the signal revealed at different distinguish rate. It was also known that the EMD was a method of principal component analysis and the decomposed result containing the main information of the origin signals. However the decomposed IMF was not a standard IMF containing only one component but a nonstandard IMF containing many components. The phenomenon was outstanding in the first three orders IMF components. Twenty orders PNN auto regression model was used to make time-varying parameter analysis to the first three orders IMF components and the time-varying auto-spectral density were obtained respectively, as the following fig3, fig4 and fig5. The energy of vibration signals mainly concentrated in the range from $230 \mathrm{~Hz}$ to $260 \mathrm{~Hz}$. The change trend of the 
energy with the time was clearly displayed in this range. The conclusion that the vibration signals and the stimulation signals had much pertinence in the range obtained. Considered the relation between response and stimulation in kinetics system, the energy of stimulation signals mainly concentrated in this range also and the dynamic variation was also the same with the vibration response. The energy of signal at $130 \mathrm{~Hz}$ was strong at beginning but decreased with time. Hence the signal at $130 \mathrm{~Hz}$ might be disturbing signal. The energy of signal at $40 \mathrm{~Hz}$ was relatively weak and was not dynamic variation throughout the time. Hence the signal at $40 \mathrm{~Hz}$ might be a structure signal or a load signal

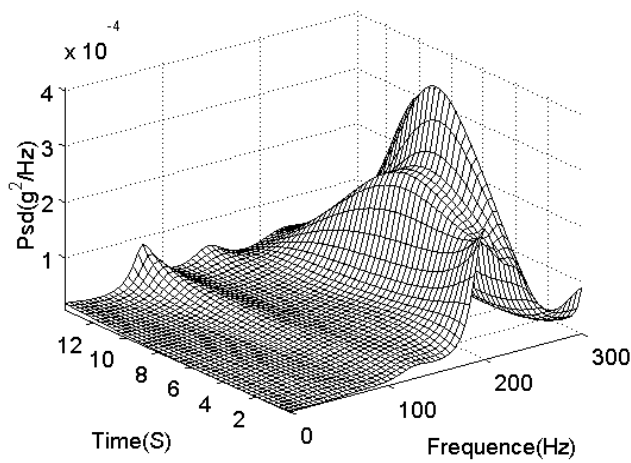

Fig.3: PNN time-frequency spectrum of 1thorder IMF.

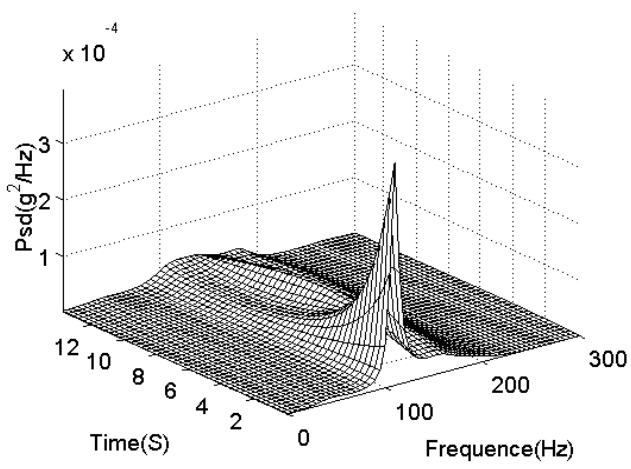

Fig.4:PNN time-frequency spectrum of 2ndorder IMF.

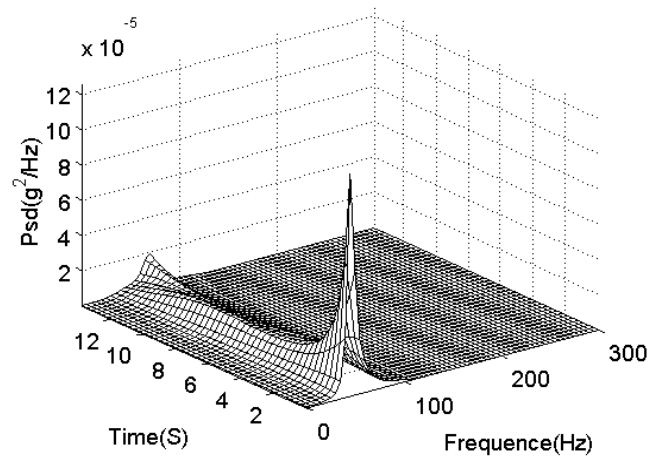

Fig.5:PNN time-frequency spectrum of 3rd order IMF.

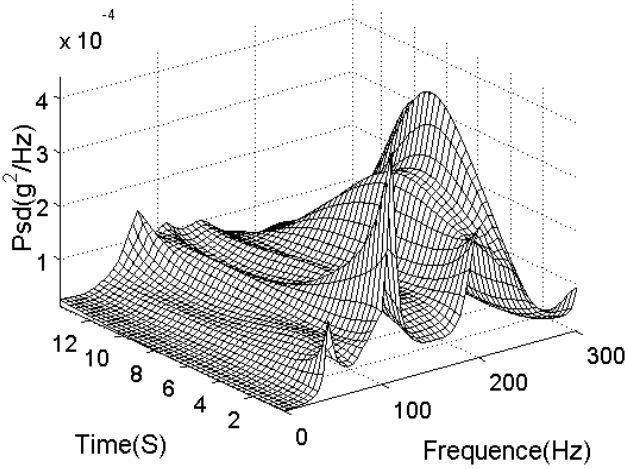

Fig.6:PNN time-frequency spectrum of all IMFs.

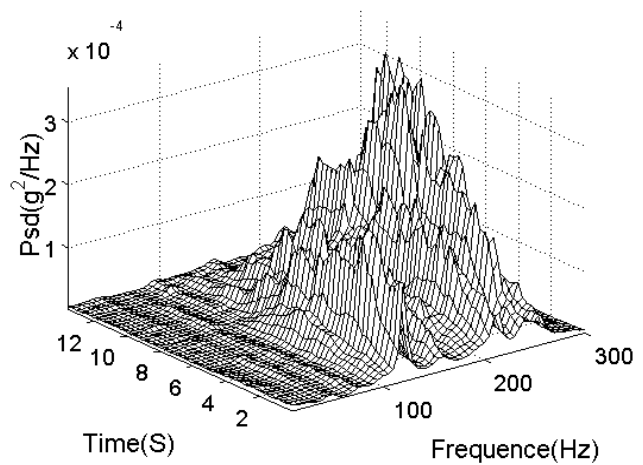

Fig.7:STFT time-frequency spectrum which has little pertinence with stimulation of this system.

The time-varying auto-spectral density could be reconstituted by linear superposing through the time-varying auto-spectral density of the first three order IMFs. As in the following fig6. A comparation between the power spectrum in fig6 and in fig7 obtained by the method of short-time Fourier transform had been made. It showed that the two spectrums both described the time-frequency character of signals realistically. Moreover the law of energy changing with time was also same. But there existed disturbance of the crossed signals and the distinguish rate of frequency in fig11 was much less clear than in fig10 .Hence the method to estimate time-varying $A R$ model parameter and time-varying auto-spectral density by PNN is valid. The method avoids the correlative estimate by using the datum directly. So the method has the characters of less-workload and high-precision distinguish rate of frequency. Besides it can analyze a large-datum signal with less EMS memory by modulating the number of hide layers and neural cell according to real occasion.

\section{Conclusions}


The results indicate that it is perfectly to analyze some nonlinear amplitude modulation signals whose frequencies are very close through multi-component PPN based on EMD. The method has avoided greatly the inconsistency brought by the approximation of taking multi-component IMF as single-component IMF. Hence the method has improved distinguish rate of frequency and reduced random error.

\section{References}

[1] G.S. Hu, Digital signal processing: theory, $m$ -ethod and realizing. Tsinghua University $\mathrm{Pr}$ -ess, Beijing, 2003.

[2] N..E. Huang, Z. Shen. and S..R. Long., The empirical mode decomposition and the Hilbert spectrum for nonlinear and nonstationary time-series analysis. ProcR Soc, 454:903-99 5, 1998.

[3] Y.M.. Zhong, S.R.. Qin and B.P.. Tang, Study on a new transform method forvibration si gnal. Journal of Vibration Engin-eering, 15:2 33-238, 2002.

[4] J.S.. Cheng, D.J.. Yu and Y. Yang, R-esearch on the intrinsic mode function(IMF)criterion in EMD method. Mechanical Syste-ms and Signal Processing, 20:817-824, 2006.

[5] Z.W.. Liu and X.Q. Lian, Research andapplic ation of artificial intelligence for waterenviro nment protection. Procedings of 2006 Intern ational Conference on AI(ICAI'06), pp.153-1 56, 2006.

[6] Z.. Chen and S.X. Zheng, Analysis on end e ffects of EMD method. Journal ofData Acq uisition \& Processing, 18:114-118, 2003.

[7] X.G. He and J.Z.. Liang, Process neur-al net work. World Computer Congress 2000,Proce edings of Conference on Intelligent Inf-orma tion Processing, pp.143-146, 2000.

[8] S.H.. Xu and X.G.. He, Research and applica tions of radial basis PNN. Journal ofBeijing University of Aeronautics and Astro-nautics, 30:14-17, 2004.

[9] F. Konzin. and F. Nakajima, The order deter -mination problem for linear time-varying A Rmodels. IEEE Transon Automatic Control, 2 5 :250-257, 1980. 